Bioagro 33(1): 59-64. 2021

doi: http://www.doi.org/10.51372/bioagro331.8

NOTA TÉCNICA

\title{
EFECTO DEL PACLOBUTRAZOL SOBRE EL CRECIMIENTO DE LA PLANTA, RENDIMIENTO Y CALIDAD DEL FRUTO EN TOMATE
}

\author{
Josúe Ramos-Fernández ${ }^{1}$, Oscar J. Ayala-Garay², Mario Pérez-Grajales ${ }^{1}$, \\ Felipe Sánchez-del Castillo ${ }^{1}$ y J. Jesús Magdaleno-Villar ${ }^{1}$
}

\begin{abstract}
RESUMEN
Existen pocos estudios del efecto del retardador de crecimiento Paclobutrazol (PBZ) en el ciclo completo de producción del tomate cultivado en invernadero. En esta investigación se evaluó el efecto en variables vegetativas y reproductivas del híbrido de crecimiento indeterminado El Cid. Se evaluaron cinco tratamientos que variaron de cero a cuatro aplicaciones (a los $25,77,92$ y 107 días después de la siembra), con dosis de $50 \mathrm{mg} \cdot \mathrm{L}^{-1}$ de PBZ (Cultar $25 \mathrm{SC}$ ) dirigidas al follaje, utilizando un diseño completamente al azar con cuatro repeticiones. La altura de planta y la longitud de los entrenudos se redujeron con relación a las plantas no tratadas, y el efecto fue mayor a medida que aumentó el número de aplicaciones. El número de entrenudos no resultó afectado. El rendimiento de frutos se incrementó $(P \leq 0,05)$ cuando las plantas recibieron cuatro aplicaciones, al presentar aproximadamente 30 frutos con 3,573 kg de peso total por planta, y en las no tratadas 26 frutos y 3,078 kg por planta. El peso promedio de fruto, el grosor del pericarpio y el contenido de sólidos solubles fueron similares en las plantas tratadas y no tratadas. Con aplicaciones hasta la formación el quinto racimo floral, un total de cuatro aplicaciones resultaron las más eficientes en la reducción de la altura de planta y mejorar el rendimiento por planta, sin afectar la calidad del fruto.
\end{abstract}

Palabra clave adicionales: Altura de planta, calidad del fruto, PBZ, retardantes de crecimiento

\begin{abstract}
Effect of paclobutrazol on plant growth, yield and fruit quality in tomato

There are few studies on the effect of the growth retardant Paclobutrazol (PBZ) on the entire tomato production cycle. In this research, vegetative and reproductive variables of the plant were studied in the indeterminate hybrid El Cid under greenhouse. Five treatments were evaluated from zero to four applications (25, 77, 92 and 107 days after sowing), with doses of $50 \mathrm{mg} \cdot \mathrm{L}^{-1}$, in a completely randomized design with four repetitions using ten plants as the experimental unit. The PBZ (Cultar 25 SC) was applied to the foliage. Plant height and length of the internodes were reduced in relation to the untreated plants, and the effect was greater as the number of applications increased. The number of internodes was not affected. The fruit yield increased $(P \leq 0.05)$ when the plants received four applications, presenting approximately 30 fruits with $3,573 \mathrm{~kg}$ of total weight per plant, and in the untreated 26 fruits and 3,078 kg per plant. Average fruit weight, pericarp thickness, and soluble solids content were similar in treated and untreated plants. With applications up to the fifth flowering, a total of four applications were the most efficient in reducing plant height and improving yield per plant, without affecting the quality of the fruit.
\end{abstract}

Additional keywords: Fruit quality, growth retardants, PBZ, plant height

\section{INTRODUCCIÓN}

De acuerdo con FAOSTAT (2018), México es el noveno país productor de tomate fresco del mundo con 4,5 millones de toneladas. El sistema de producción convencional de tomate se caracteriza por prácticas de manejo de un tallo por planta, con eliminación (poda) de brotes laterales

Recibido: Mayo 17, 2020

Aceptado: Noviembre 16, 2020

${ }^{1}$ Dpto. de Fitotecnia, Universidad Autónoma Chapingo. Chapingo, estado de México, México. e-mail: josue.r.f.91@gmail.com; perezgm7@yahoo.com.mx; fsanchezdelcastillo@yahoo.com.mx; jmagdalenovillar8@gmail.com (autor de correspondencia).

${ }^{2}$ Programa de RGP-Fisiología Vegetal. Colegio de Posgraduados, Montecillo, México. e-mail: oayala@colpos.mx 
y hojas, en ciclos de cultivo largos (Santos y Sánchez, 2003). Con ello, los rendimientos por hectárea llegan a alcanzar hasta 400 toneladas en un año (Nuez, 2001), aunque los costos de producción son muy elevados (Méndez et al., 2005).

Una forma alternativa de incrementar la producción de tomate por unidad de superficie en invernadero, es promover la formación y crecimiento de mayor número de flores y frutos por racimo (Bernier y Perilleux, 2005). Esto puede ser influenciado por aplicaciones exógenas de reguladores de crecimiento, que afecten las relaciones fuente-demanda de los órganos en crecimiento en etapas tempranas, lo que puede mejorar la uniformidad después del trasplante e incrementar la diferenciación de flores en los primeros racimos (Chen et al., 2020).

El Paclobutrazol (PBZ) es un retardante del crecimiento de las plantas y fungicida del grupo de los triazoles. Su principal modo de acción es la inhibición de la biosíntesis de las giberelinas (Jankiewicz, 2003). De esta manera, retarda el crecimiento vegetativo, incrementa el crecimiento de las raíces, reduce el crecimiento de los entrenudos del tallo, induce la formación de yemas florales y produce mejor calidad de fruta en diversos cultivos. También estimula la precocidad e incrementa la formación de semillas en el fruto de tomate (Berova y Zlatev, 2000).

La respuesta de las plantas de tomate está en función del tipo de regulador, concentración y método de aplicación, así como del estado fenológico de la planta (Fletcher et al., 2000).

En diversas investigaciones se ha evaluado el efecto del PBZ aplicado en semillas (Campos et al., 2014) o plántulas de tomate (Berova y Zlatev, 2000; Ferreira et al., 2017), pero existen muy pocos estudios sobre aplicaciones durante el ciclo completo de producción sobre el rendimiento y la calidad del fruto (Chen et al., 2020). Por ello, el objetivo del presente estudio fue la evaluación del PBZ asperjado al follaje, desde plántula hasta la aparición del quinto racimo floral, para determinar su efecto sobre variables vegetativas $y$ reproductivas en el híbrido de tomate El Cid, de crecimiento indeterminado.

\section{MATERIALES Y MÉTODOS}

El estudio se efectuó en 2017 en un invernadero de $6 \mathrm{~m}$ de altura, cubierto con doble capa de polietileno blanco difusor de luz, ubicado en Chiconcuac, México, a 19³2' N, 9852' W, y 2246 msnm de altitud. La siembra se realizó el 14 de enero en contenedores de poliestireno de 200 cavidades, con turba como sustrato.

El material vegetal empleado fue el híbrido El Cid F1 (Harris Moran, México), el cual es un híbrido de crecimiento indeterminado y fruto tipo saladette. El trasplante se hizo en suelo un mes después de la siembra; lo cual se realizó en camas de cultivo de 0,3 $\mathrm{m}$ de altura, 0,8 $\mathrm{m}$ de ancho $\mathrm{y}$ distanciadas $0,8 \mathrm{~m}$, cubiertas con una lámina de polietileno blanco impermeable. Se establecieron dos hileras de cultivo por cama, con una distancia de $0,35 \mathrm{~m}$ entre hileras y $0,3 \mathrm{~m}$ entre plantas, lo que aseguró una densidad de 4,17 plantas $\cdot \mathrm{m}^{-2}$. En éstas se colocaron dos cintillas de goteo autocompensadas, separadas a $35 \mathrm{~cm}$, con goteros cada $30 \mathrm{~cm}$. Se mantuvo la tensión de humedad del suelo en valores cercanos a 10 cb (80\% de humedad aprovechable), controlada mediante tensiómetros colocados a $15 \mathrm{~cm}$ de profundidad. Se aplicó fertilización con macro y micronutrimentos basada en recomendaciones para el cultivo de tomate en invernadero.

Las plantas se condujeron a un solo tallo, sostenidas del alambre de tutoreo a $4 \mathrm{~m}$ de altura. Una vez que la planta iba alcanzando una altura superior a $3 \mathrm{~m}$, porciones crecientes de la base del tallo eran colocadas horizontalmente sobre la cama. De esta forma, la longitud total alcanzada por el tallo fue considerada como la altura de planta Durante la floración, se introdujeron colmenas de abejorros Bombus terrestris para garantizar una adecuada polinización.

Se evaluaron cinco tratamientos (Cuadro 1). Se empleó el producto comercial Cultar 25 SC (Syngenta, México), el cual contiene $250 \mathrm{~g} \cdot \mathrm{L}^{-1}$ del ingrediente activo PBZ. Las aspersiones se realizaron con un atomizador manual, dirigidas al follaje de la planta hasta humedecerla por completo y procurando hacer el mismo número de atomizaciones en cada aplicación.

Variables respuesta. La cosecha de frutos se realizó de forma manual, cuando éstos habían cambiado su coloración en al menos 50 \% (fase 5). Se realizaron cinco cortes a partir de los 92 días después del trasplante (ddt), cada 7 días, hasta el día 120 ddt. 
Cuadro 1. Descripción de los tratamientos, momento de aplicación y concentraciones de Paclobutrazol en el híbrido de tomate El Cid cultivado en invernadero

\begin{tabular}{cccc}
\hline Tratamiento & $\begin{array}{c}\text { Número de } \\
\text { aplicaciones }\end{array}$ & $\begin{array}{c}\text { Aplicación } \\
\text { (días después de la siembra) }\end{array}$ & $\begin{array}{c}\text { Dosis } \\
\left(\mathrm{mg} \cdot \mathrm{L}^{-1} \text { ) }\right.\end{array}$ \\
\hline T0 & Sin aplicación & Sin aplicación & 0 \\
T1 & 1 & 25 & 50 \\
T2 & 2 & $25+77$ & 50 \\
T3 & 3 & $25+77+92$ & 50 \\
T4 & 4 & $25+77+92+107$ & 50 \\
\hline
\end{tabular}

Se evaluaron los siguientes caracteres en los frutos:

Número, peso del fruto y rendimiento. En 10 plantas para cada corte, así como para el total de los cinco cortes.

Sólidos solubles totales (SST). A partir de nueve frutos por tratamiento y repetición se determinó la cantidad de sólidos solubles en el jugo, empleando un refractómetro digital Atago PR-100.

Grosor de pericarpio. Medido en la zona ecuatorial del fruto, utilizando un vernier, en los mismos nueve frutos anteriores. A los $120 \mathrm{ddt}$, además de las variables anteriores, se emplearon 10 plantas para evaluar los siguientes caracteres:

Altura de planta. Se midió la longitud del tallo, desde el cuello hasta el ápice de la planta.

Número de entrenudos. Se contó el número de entrenudos. Adicionalmente, este valor permitió obtener su longitud promedio a partir de la longitud total del tallo.

Se empleó un diseño experimental completamente al azar con 5 tratamientos, 4 repeticiones y 10 plantas como unidad experimental. En total, el ensayo constó de 200 plantas establecidas en el centro de dos camas continuas en la mitad sur del invernadero, en una superficie de $48 \mathrm{~m}^{2}$.

Para el estudio de los datos se realizaron análisis de varianza y pruebas de comparación de medias de Tukey, apoyados en el programa de análisis estadístico SAS v. 9.0 (Cary, NC, USA).

\section{RESULTADOS Y DISCUSIÓN}

Caracteres vegetativos. Se encontraron diferencias estadísticas $(P \leq 0,05)$ entre los tratamientos ya que las plantas tratadas con PBZ presentaron menor altura que las no tratadas (Cuadro 2). Se observó que el PBZ aplicado tres y cuatro veces (tratamientos 3 y 4) redujeron más la altura de planta (promedio de 448,63 y 415,58 cm, respectivamente). El tratamiento sin aplicación (control) fue el de mayor altura $(551,22 \mathrm{~cm})$ de los 5 tratamientos. La reducción de altura fue de 9, 11,19 y $25 \%$ cuando se asperjó una, dos, tres o cuatro veces, respectivamente, con relación a las plantas no tratadas. Al respecto, Campos et al. (2014) mencionan que el Paclobutrazol aplicado a la semilla, con dosis de 38,5, 76,9 y $115,4 \mathrm{mg} \cdot \mathrm{L}^{-1}$, provocó reducción de la altura en 10, 21 y $24 \%$, respectivamente, con relación al tratamiento sin aplicación en plántulas de tomate. En el cultivo de papa, Flores et al. (2016) observaron que dos aplicaciones semanales de Paclobutrazol, con dosis de $1 \mathrm{mg} \cdot \mathrm{L}^{-1}$ de los 22 a los 85 días después del trasplante redujeron la altura de la planta en un $20 \%$. Estos resultados coinciden a lo señalado por otros autores (Ferreira et al., 2017) respecto a que las aplicaciones foliares de PBZ son efectivas en la reducción de la altura de planta de tomate.

En cuanto a la variable longitud de entrenudos, se encontró diferencia significativa $(P \leq 0,05)$ entre los tratamientos (Cuadro 2). Al igual que la variable anterior, las plantas asperjadas con PBZ presentaron menor longitud de entrenudos que las no tratadas, también se observó que a mayor número de aplicaciones la longitud del entrenudo se acortó en $0,71 \mathrm{~cm}(8 \%)$ con una aplicación, $1,12 \mathrm{~cm}$ (12\%) con dos aplicaciones, $1,72 \mathrm{~cm}$ (19\%) con tres aplicaciones y 2,09 cm (23\%) con cuatro aplicaciones, comparadas con las plantas del tratamiento control.

En ese sentido, el PBZ, al incidir en la vía metabólica de síntesis de giberelinas, fitohormonas encargadas de promover la división y el alargamiento celular, causa como respuesta morfológica acortamiento de entrenudos y disminución en la altura de la planta (Jankiewicz, 2003; Taiz et al. 2015). Así, por ejemplo, Seleguini et al. (2016) mencionan que una aplicación de Paclobutrazol con dosis de 
$100 \mathrm{mg} \cdot \mathrm{L}^{-1}$ a los 15 días después de la siembra en el híbrido de tomate AF 7631, tuvo una reducción en la longitud de planta de 55, 44, 77 y
$15 \%$ respecto al control sin aplicación en evaluaciones realizadas a los 17, 34, 51 y 65 ddt, respectivamente.

Cuadro 2. Altura de la planta (longitud del tallo) y número de entrenudos por planta en el híbrido de tomate El Cid, ante diferente número de aplicaciones de Paclobutrazol, en condiciones de invernadero

\begin{tabular}{clcc}
\hline & Tratamiento & Altura de planta $(\mathrm{cm})$ & Longitud de entrenudos $(\mathrm{cm})$ \\
\hline T0 & Sin aplicación (control) & $551,22 \mathrm{a}$ & $9,08 \mathrm{a}$ \\
T1 & Una aplicación & $501,81 \mathrm{~b}$ & $8,37 \mathrm{~b}$ \\
T2 & Dos aplicaciones & $490,67 \mathrm{~b}$ & $7,96 \mathrm{c}$ \\
T3 & Tres aplicaciones & $448,63 \mathrm{c}$ & $7,36 \mathrm{~d}$ \\
T4 & Cuatro aplicaciones & $415,58 \mathrm{c}$ & $6,99 \mathrm{e}$ \\
\hline
\end{tabular}

Medias con diferente letra entre columnas son estadísticamente diferentes, de acuerdo con la prueba de Tukey $(P \leq 0,05)$

Los resultados de la presente investigación, al igual que en otros trabajos, demuestran el efecto del PBZ, como retardante de crecimiento, sobre la altura de plantas de tomate. El beneficio en la producción se refleja en plantas más compactas con menor competencia entre ellas, además de facilitar el manejo de la planta y la cosecha por la menor altura, sobre todo en los cultivares de hábito de crecimiento indeterminado, como fue el caso.

El número de entrenudos presentó valores muy similares entre sí, con un promedio de 60,53 entrenudos a lo largo del tallo de la planta. Los retardadores inhiben el alargamiento de los entrenudos, aunque el número de ellos, por lo general, no cambia (Jankiewicz, 2003).

Componentes del rendimiento. Un componente fundamental en el rendimiento de tomate es el número de frutos por planta, ya que de este carácter depende la expresión máxima de la producción (Sánchez et al., 2014). Este componente está determinado por el número de flores que son fecundadas y alcanzan a desarrollarse como fruto. Se encuentra influenciado por la relación fuente-demanda en diferentes fases del ciclo de vida de la planta (Santiago et al., 1998). En la presente investigación, donde los tratamientos 2 y 3 resultaron estadísticamente diferentes $(P \leq 0,05)$ entre ellos en la variable número de frutos por planta (Cuadro 3); únicamente se observaron diferencias significativas $(P \leq 0,05)$ al considerar el número total de frutos por planta de los tratamientos extremos (T0 y T4). El tratamiento 4, es decir, el de mayor número de aplicaciones, presentó la mayor cantidad de frutos cosechados por planta con un promedio de 29,8 en contraste con 26 del tratamiento sin aplicación de PBZ, esto es, $15 \%$ más, lo que podría ser relevante desde el punto de vista de rentabilidad. De acuerdo con Carvalho et al. (2019) al realizar aplicaciones de $42,5 \mathrm{mg} \cdot \mathrm{L}^{-1}$ de $\mathrm{PBZ}$, asperjado en plántulas de 15 días después de emergidas, se incrementó el número de flores por inflorescencia, en plantas de tomate del híbrido N901; en ese sentido, en el presente estudio el amarre o cuajado de frutos se incrementó con las aplicaciones del retardante.

Se detectaron diferencias significativas $(P \leq 0,05)$ para la variable rendimiento por planta (Cuadro 3). Se observa que el tratamiento con cuatro aplicaciones de PBZ alcanzó el valor más alto con un promedio de $3,573 \mathrm{~kg}$ por planta en comparación con $3,078 \mathrm{~kg}$ por planta del tratamiento sin aplicación, es decir $16 \%$ de incremento. Resultados similares fueron hallados en la investigación de Carvalho et al. (2019) quienes encontraron un incremento de 27,2 \% del rendimiento.

A diferencia de esta investigación, Ferreira et al. (2017) no hallaron efecto sobre el rendimiento y número de frutos con el uso de PBZ en etapas de plántula, en la tercera y quinta hoja. Pero en nuestro caso, las aplicaciones se hicieron a lo largo del ciclo de cultivo, a partir de la etapa de plántula hasta la aparición del quinto racimo floral lo que habría permitido una respuesta favorable en la planta. 
Cuadro 3. Número total de frutos y rendimiento total por planta, en cinco cortes, en el híbrido de tomate El Cid, ante diferente número de aplicaciones de Paclobutrazol, en condiciones de invernadero

\begin{tabular}{clcc}
\hline & Tratamiento & Número total de frutos & Rendimiento por planta $(\mathrm{kg})$ \\
\hline T0 & Sin aplicación & $259,68 \mathrm{~b}$ & $3,078 \mathrm{~b}$ \\
T1 & Una aplicación & $268,00 \mathrm{ab}$ & $3,155 \mathrm{ab}$ \\
T2 & Dos aplicaciones & $279,68 \mathrm{ab}$ & $3,381 \mathrm{ab}$ \\
T3 & Tres aplicaciones & $285,00 \mathrm{ab}$ & $3,413 \mathrm{ab}$ \\
T4 & Cuatro aplicaciones & $298,00 \mathrm{a}$ & $3,573 \mathrm{a}$ \\
\hline
\end{tabular}

Medias con diferente letra entre columnas son estadísticamente diferentes, de acuerdo con la prueba de Tukey $(P \leq 0,05)$

El peso del tomate fue muy similar en todos los tratamientos con un promedio general de 119,36 g por fruto. Es decir, los frutos mantuvieron similar peso y tamaño ya sea que las plantas fuesen asperjadas o no con el producto. Bernier y Perilleux (2005) señalan que esta variable depende de la cantidad de fotoasimilados disponibles para su crecimiento, y Taiz et al. (2015) destacan que el peso del fruto es el resultado de la relación entre la actividad de la fuente y la fuerza de la demanda durante el periodo de crecimiento, donde se involucran de manera importante las características del genotipo.

Ferreira et al. (2017) observaron que el efecto del retardante se va perdiendo con el tiempo. En función de esto, se desconoce si al continuar aplicando el producto se mantendría el incremento en el rendimiento o si ello ocasionaría algún detrimento de la planta por el estrés provocado por las constantes aplicaciones.

Calidad del fruto. El análisis de la varianza no detectó diferencias en las variables de SST y grosor de pericarpio $(P>0,05)$. Los valores medios fueron de $3,9{ }^{\circ}$ Brix y $1,0 \mathrm{~cm}$, respectivamente, sin respuesta a la aplicación de PBZ. Este valor de SST se encuentra dentro del rango reportado para tomate tipo saladette y representa una buena calidad de fruto. Por ejemplo, García et al. (2010) señalan que los valores normales se encuentran entre 3,5 y 5,0 ${ }^{\circ}$ Brix. Por su parte, el grosor de pericarpio que hallamos es similar al reportado para este tipo tomate cultivado en invernadero $(0,8-0,9 \mathrm{~cm})$, lo cual se relaciona con una mayor vida postcosecha.

\section{CONCLUSIONES}

El retardador de crecimiento Paclobutrazol redujo la altura de planta, a mayor número de aplicaciones esta fue menor. El número de entrenudos no fue afectado por las aplicaciones del producto.

El empleo de cuatro aplicaciones aumentó el número de frutos totales y el rendimiento total por planta, sin afectar el tamaño de los frutos hasta el quinto racimo, ni los componentes de calidad (sólidos solubles totales y grosor de pericarpio).

\section{LITERATURA CITADA}

1. Bernier G. y C. Perilleux. 2005. A physiological overview of the genetics of flowering time control. Plant Biotechnology Journal 3(1): 3-16.

2. Berova, M. y Z. Zlatev. 2000. Physiological response and yield of paclobutrazol treated tomato plant (Lycopersicon esculentum Mill.). Plant Growth Regulation 30(2): 117-123.

3. Campos de Melo A.P., A. Seleguini, S. Da Rocha y V. Veloso. 2014. Peliculização de sementes de tomate associada ao paclobutrazol. Bragantia 73(2): 123-129.

4. Carvalho F.J., L.B. Carneiro, C.G.S. Benett, K.S.S. Benett, A.S. Martins, A.T. Silva y A. Seleguini. 2019. Plant density and application of growth regulator to tomato crop for industrial processing. Revista Colombiana de Ciencias Hortícolas 13(3): 397-405.

5. Chen S., X. J. Wang, G. F. Tan, W. Q. Zhou y G. L. Wang. 2020. Gibberellin and the plant growth retardant Paclobutrazol altered fruit shape and ripening in tomato. Protoplasma 257(3): 853-861.

6. FAOSTAT (Food and Agriculture Organization of de United Nations). 2018. The Food and Agriculture Organization Corporate Statistical Database. Tomato statistics. FAO, Roma, Italy http://www.fao.org/faostat/en/\# data/QC (consulta de mayo 7, 2020).

7. Ferreira N., E. Vendruscolo, A. Seleguini, W. 
Dourado, C. Benett y A. Nascimento. 2017. Crescimento, produção e qualidade de frutos de tomateiro em cultivo adensado com uso de paclobutrazol. Revista Colombiana de Ciencias Hortícolas 11(1): 72-79.

8. Fletcher R.A., A. Gilley, T.D. Davis y N. Sankhla. 2000 Triazoles as plant growth regulators and stress protectants. Horticultural Reviews 24(1): 55-138.

9. Flores-López R., R. Martínez-Gutiérrez, H. A. López-Delgado y M. Marín-Casimiro. 2016. Aplicación periódica de bajas concentraciones de paclobutrazol y ácido salicílico en papa en invernadero. Revista Mexicana de Ciencias Agrícolas 7(5): 1143-1154.

10. García A., A. Contreras, M. Rodríguez y N.Y. Trujillo. 2010. Características físicas y químicas del tomate (Solanum lycopersicum L.) variedad pera. @Limentech Ciencia y Tecnología Alimentaria 8(1): 75-82.

11.Jankiewicz L.S. 2003. Reguladores de Crecimiento, Desarrollo y Resistencia en Plantas. Vol. I. Propiedades y Acción. Universidad Autónoma Chapingo. Ed. Mundiprensa. México, D.F. 487 p.

12. Méndez G.T., F. Sánchez-del Castillo, C.J. Sahagún y M.E. Contreras. 2005. Doseles escaleriformes con hileras de plantas de jitomate orientadas en dirección este-oeste. Revista Chapingo Serie Horticultura 11(1): 185-192.
13. Nuez F. 2001. El Cultivo del Tomate. Ediciones Mundiprensa. Madrid.

14. Sánchez-del Castillo F., O.A. Bastida-Cañada, E.C. Moreno-Pérez, E. Contreras-Magaña y J. Sahagún-Castellanos. 2014. Rendimiento de jitomate con diferentes métodos de cultivo hidropónico basados en doseles escaleriformes. Rev. Chapingo Serie Horticultura 20(3): 239251.

15. Santiago J., M. Mendoza y F. Borrego. 1998. Evaluación de tomate (Lycopersicon esculentum Mill) en invernadero: criterios fenológicos y fisiológicos. Agronomía Mesoamericana 9(1): 59-65.

16. Santos M.J. y F. Sánchez-del Castillo. 2003. Densidades de población, arreglos de dosel y despunte en tomate (Lycopersicon esculentum Mill.) cultivado en hidroponía. Revista Fitotecnia Mexicana 26(2): 257-262.

17. Seleguini A., E. Pradi-Vendruscolo, L. Cardoso-Campos y M. Faria Júnior. 2016. Efeito do paclobutrazol sobre o crescimento de plantas e produzção de tomate (Solanum lycopersicum L.) em ambiente protegido. Scientia Agropecuaria 7(4): 391399.

18. Taiz L., E. Zeiger, I.M. Møller y A. Murphy. 2015. Plant Physiology and Development. $6^{\text {th }}$ Ed. Sinauer Associates Publishers. Sunderland, Massachusetts, USA. 